\title{
Studies of NAD kinase and NMN:ATP adenylyltransferase in Haemophilus influenzae
}

\author{
Ana Denicola-Seoane and Bruce M. Anderson*
}

Department of Biochemistry and Nutrition, Virginia Polytechnic Institute and State University, Blacksburg, Virginia 24061-0308, USA

(Received 30 August 1989; revised 13 November 1989; accepted 21 November 1989)

\begin{abstract}
Previous studies of Haemophilus influenzae documented the importance of several pyridine nucleotide-dependent enzymes in processing extracellular NAD and NMN to satisfy the V-factor growth requirement of the organism. The substrate specificities of two of these enzymes, NMN:ATP adenylyltransferase and NAD kinase, were investigated following partial purification. The ability of the transferase to utilize 3-acetylpyridine mononucleotide and 3-aminopyridine mononucleotide as substrates for the synthesis of the corresponding dinucleotides was demonstrated. The NAD kinase was observed to accept 3-acetylpyridine adenine dinucleotide as a substrate but failed to utilize 3-aminopyridine adenine dinucleotide. The mononucleotides of 3-acetylpyridine and 3-aminopyridine were shown to be as effective as the corresponding dinucleotides in the support of growth and inhibition of growth of $\boldsymbol{H}$. influenzae, respectively. Inhibition of growth of $\boldsymbol{H}$. influenzae by submicromolar 3-aminopyridine adenine dinucleotide was shown to occur because 3-aminopyridine mononucleotide was produced from it in reactions catalysed by the $\boldsymbol{H}$. influenzae periplasmic nucleotide pyrophosphatase. The presence of an additional important pyridine nucleotide-dependent enzyme, NMN glycohydrolase, is also reported.
\end{abstract}

\section{Introduction}

Haemophilus influenzae is representative of a number of Haemophilus species that exhibit unique growth requirements for intact NAD (V-factor) and haemin (X-factor). $H$. influenzae type $\mathrm{b}$ is the primary cause of bacterial meningitis in infants and is responsible for 12000 cases annually in the USA (Givner et al., 1989). Other related Haemophilus species have been implicated in numerous diseases in humans and other animals. Treatment of $\boldsymbol{H}$. influenzae infections has relied upon the use of ampicillin and chloramphenicol; however, a continuing increase in strains resistant to these antibiotics has been observed (Gunn et al., 1974; Syriopoulou et al., 1978; Doern et al., 1986). The inability of V-factor-requiring organisms to synthesize NAD from low molecular mass precursor molecules utilized by other bacterial systems suggested the possible presence of processes of NAD metabolism unique to these organisms, the inhibition or regulation of which might provide an alternative approach to controlling growth of these organisms. Recent studies of the

\footnotetext{
Abbreviations: AAD, 3-aminopyridine adenine dinucleotide; AMN, 3-aminopyridine mononucleotide; APAD, 3-acetylpyridine adenine dinucleotide; APMN, 3-acetylpyridine mononucleotide.
}

utilization of externally-added intact NAD for growth of $H$. influenzae revealed the presence of a periplasmic nucleotide pyrophosphatase (EC 3.2.2.6) responsible for the hydrolysis of NAD, providing nicotinamide mononucleotide for the intracellular re-synthesis of NAD (Kahn \& Anderson, 1986). An effective inhibition of growth by ADP observed in these studies correlated well with the selective inhibition of the nucleotide pyrophosphatase by ADP. 3-Acetylpyridine adenine dinucleotide, an NAD analogue known to serve as a coenzyme for a variety of dehydrogenases, was an effective substrate for the nucleotide pyrophosphatase and was demonstrated to support growth of $H$. influenzae in the absence of NAD. Another substrate for the nucleotide pyrophosphatase, 3-aminopyridine adenine dinucleotide, which has not been observed to function with dehydrogenases, was a potent inhibitor of growth of $H$. influenzae, causing significant growth inhibition at $0.2 \mu \mathrm{M}$ (Kahn \& Anderson, 1986). These studies raised a number of questions as to the fate of the mononucleotides of these two NAD analogues produced through the activity of the periplasmic nucleotide pyrophosphatase. In order for the 3-acetylpyridine mononucleotide to satisfy the V-factor growth requirement of the organism, intracellular conversion to the NAD and NADP 
analogues should occur; however, little is known about the substrate specificities of the cytoplasmic enzymes involved, the NMN:ATP adenylyltransferase (EC 2.7.7.1) and NAD kinase (EC 2.7.1.23). Also, understanding the interactions of 3-aminopyridine mononucleotide with these enzymes could aid in the elucidation of the mechanism of the growth inhibition by submicromolar concentrations of 3-aminopyridine adenine dinucleotide.

Studies of the interactions of pyridine nucleotide analogues with the partially purified NMN:ATP adenylyltransferase and NAD kinase presented here provide new information concerning the functioning of these analogues with $\boldsymbol{H}$. influenzae. In addition, a new catalytic activity, NMN glycohydrolase (EC 3.2.2.14), has been demonstrated in $\boldsymbol{H}$. influenzae.

\section{Methods}

Growth of the organism. Haemophilus influenzae strain $\mathrm{Rd}$ was obtained from Dr William L. Albritton of the University of Saskatchewan, Saskatoon, Canada. Studies to investigate the ability of compounds to support or inhibit growth of $H$. influenzae were carried out in Brain Heart Infusion media as described previously (Kahn \& Anderson, 1986). Growth rates were determined turbidimetrically by measuring $\mathrm{OD}_{660}$. The rate of growth was expressed as doubling time values, measured as the time required for the $\mathrm{OD}_{660}$ to increase from $0 \cdot 2$ to $0 \cdot 4$. Growth rates are reported as means of three separate growth studies, in which growth rate varied within $\pm 10 \%$. For the purpose of enzyme purification, $\boldsymbol{H}$. influenzae cells were harvested after $10 \mathrm{~h}$ (late linear phase), washed and stored at $-15^{\circ} \mathrm{C}$ in a minimal amount of $50 \mathrm{~mm}$-potassium phosphate, $\mathrm{pH} 7 \cdot 0$.

Synthesis of pyridine mononucleotides. 3-Acetylpyridine mononucleotide and 3-aminopyridine mononucleotide were prepared from 3acetylpyridine adenine dinucleotide and 3-aminopyridine adenine dinucleotide, respectively, as described by Christ \& Cooper (1980), and purified by ion-exchange chromatography on Dowex-1-formate. Purity of the mononucleotides was determined using high performance liquid chromatography (HPLC) and thin layer chromatography techniques.

Partial purification of NMN:ATP adenylyltransferase. Frozen cells $(20 \mathrm{~g})$ were dispersed at $4{ }^{\circ} \mathrm{C}$ to a final volume of $100 \mathrm{ml}$ using $50 \mathrm{mM}$ potassium phosphate, $\mathrm{pH} 7.0$, containing $0.5 \mathrm{~mm}$-DTT, using a glass homogenizer. The resulting suspension was sonicated in an ice-salt bath for $5 \mathrm{~min}$ and cell debris removed by centrifugation for $20 \mathrm{~min}$ at $17000 \mathrm{~g}$. Protamine sulphate $(2 \%, \mathrm{w} / \mathrm{v}$, solution) was added to the sonicate supernatant at $4{ }^{\circ} \mathrm{C}$ to give a final concentration of $0.2 \%$. After stirring for $30 \mathrm{~min}$, the mixture was centrifuged at $39000 \mathrm{~g}$ for $10 \mathrm{~min}$ and the resulting supernatant was dialysed against 3 litres $20 \mathrm{mM}$ potassium phosphate, $\mathrm{pH} 7.5$, containing $0.5 \mathrm{~mm}$-DTT. The dialysed fraction was applied to a Matrex gel Green A column $(1 \times 10 \mathrm{~cm})$ previously equilibrated at $4^{\circ} \mathrm{C}$ in $50 \mathrm{~mm}$-potassium phosphate, $\mathrm{pH} 7.5$, containing 0.5 mM-DTT. A linear salt gradient, 0.05 to $0.8 \mathrm{mM}-\mathrm{KCl}$ in the same buffer, removed some unwanted protein. The NMN : ATP adenylyltransferase was then eluted by a frontal elution with $1 \mathrm{M}-\mathrm{KCl}$ in the equilibration buffer. Fractions $(2.5 \mathrm{ml})$ containing the enzyme were pooled and concentrated by ultrafiltration. The enzyme was purified 15 -fold with a $27 \%$ yield and a final specific activity of 0.02 units (mg protein) $)^{-1}$
Partial purification of NAD kinase. A cell sonicate was prepared from $20 \mathrm{~g}$ of frozen cells as described above and centrifuged at $100000 \mathrm{~g}$ for $60 \mathrm{~min}$ at $40^{\circ} \mathrm{C}$. The resulting supernatant was applied to a Matrex gel Green A column $(1 \times 11 \mathrm{~cm})$ previously equilibrated with $10 \mathrm{~mm}$ potassium phosphate, $\mathrm{pH} 7 \cdot 0$, containing $1 \mathrm{mM}$-DTT. After washing the column with equilibration buffer, the NAD kinase was eluted by applying a linear salt gradient, 0 to $0.5 \mathrm{M}-\mathrm{KCl}$ in the same buffer. Fractions $(2.5 \mathrm{ml})$ containing NAD kinase activity were pooled, concentrated and dialysed against $10 \mathrm{~mm}$-potassium phosphate, pH 7.0, containing $1 \mathrm{~mm}$-DTT. The dialysed fraction was applied to a Matrex gel Blue A column $(1 \times 10 \mathrm{~cm})$ and NAD kinase activity was eluted by washing with equilibration buffer. The enzyme was purified 18 -fold with a $32 \%$ yield and a final specific activity of 0.05 unit (mg protein) $)^{-1}$.

Kinetic analysis of enzymes. In studies of NMN : ATP adenylyltransferase, initial velocities of NAD formation were determined in reaction mixtures $(1 \mathrm{ml})$ containing $75 \mathrm{mM}-\mathrm{Tris} / \mathrm{HCl}, \mathrm{pH} 8.0,0.5 \%$ ethanol, $30 \mathrm{mM}-\mathrm{MgCl}_{2}, 3$ units of yeast alcohol dehydrogenase, $0.2 \mathrm{ml}$ NMN : ATP adenylyltransferase $\left(0.2 \mathrm{mg}\right.$ protein $\left.\mathrm{ml}^{-1}\right)$, and varying concentrations of NMN and ATP. Ethanol and yeast alcohol dehydrogenase were included as a coupled enzyme system to assay, by reduction, any NAD formed.

The ability of NMN:ATP adenylyltransferase to use 3-acetylpyridine mononucleotide as a substrate with ATP, to form the corresponding dinucleotide, was studied in reaction mixtures $(1 \mathrm{ml})$ containing $75 \mathrm{mM}-\mathrm{Tris} / \mathrm{HCl}, \mathrm{pH} 8.0,0.5 \%$ ethanol, $2.5 \mathrm{mM}-\mathrm{ATP}$, $4.3 \mathrm{~mm}$-3-acetylpyridine mononucleotide, $30 \mathrm{~mm}-\mathrm{MgCl}_{2}$, and $0.3 \mathrm{ml}$ NMN : ATP adenylyltransferase (0.2 $\mathrm{mg}$ protein $\left.\mathrm{ml}^{-1}\right)$. In this case, 3 units of horse liver alcohol dehydrogenase were included for the coupled enzyme assay since 3-acetylpyridine adenine dinucleotide functions more effectively with the horse liver enzyme than with the yeast alcohol dehydrogenase. In reaction mixtures not containing the coupled enzyme system, the formation of 3-acetylpyridine adenine dinucleotide was confirmed by HPLC. Identification of this dinucleotide by HPLC was achieved on an ion-exchange column $(4.6 \times 250 \mathrm{~mm})$ packed with Alltech RSIL-AN resin, equilibrated and eluted with $100 \mathrm{~mm}$-potassium phosphate, $\mathrm{pH} 3 \cdot 8$. The retention time for the experimentally-produced 3-acetylpyridine adenine dinucleotide was compared with that of a known standard.

The ability of NMN : ATP adenylyltransferase to use 3-aminopyridine mononucleotide with ATP to form 3-aminopyridine adenine dinucleotide was investigated using $1.7 \mathrm{~mm}$-3-aminopyridine mononucleotide in the reaction mixtures described above in the absence of the coupled enzyme system, since 3-aminopyridine adenine dinucleotide does not serve as coenzyme for either of the alcohol dehydrogenases. The formation of this dinucleotide as catalysed by the NMN : ATP adenylyltransferase was demonstrated by the HPLC assay described above, as well as by thin layer chromatography on cellulose plates (solvent system of $95 \%$ ethanol/0.1 M-acetic acid, $1: 1, \mathrm{v} / \mathrm{v}$ ) and detection by both UV absorbance and fluorescence. With both of these analyses, the time-dependent conversion of 3-aminopyridine mononucleotide to 3-aminopyridine adenine dinucleotide was readily observed.

Kinetic parameters for NAD kinase were determined in reaction mixtures $(1 \mathrm{ml})$ containing $50 \mathrm{~mm}-\mathrm{Tris} / \mathrm{HCl}, \mathrm{pH} 8.0,7.5 \mathrm{~mm}-\mathrm{MgCl}_{2}$, $0.1 \mathrm{ml}$ partially purified NAD kinase $\left(0.1 \mathrm{mg}\right.$ protein $\left.\mathrm{ml}^{-1}\right)$, varying concentrations of NAD and ATP, with $5 \mathrm{~mm}$-glucose 6-phosphate and 0.4 unit yeast glucose 6-phosphate dehydrogenase included as a coupled enzyme assay system. Initial velocities of NADP formation were determined spectrophotometrically at $340 \mathrm{~nm}$, measuring the NADPH produced by the coupling system.

Spectrophotometric measurements were performed on a Beckman Acta MVI recording spectrophotometer. In enzyme rate studies, kinetic parameters were determined using Cleland computer programs (Cleland, 1979). Analyses by HPLC were carried out on a Spectra- 
Physics SP8000 chromatograph. Protein concentrations were determined by the microprotein assay of the Coomassie blue method (Bradford, 1976) using bovine serum albumin as a standard. Ultrafiltration was carried out using Amicon PM-10 membranes.

Chemicals. Brain Heart Infusion media and triethanolamine were obtained from Fisher Scientific. Protamine sulphate, haemin, bovine serum albumin, dithiothreitol (DTT), 3-acetylpyridine adenine dinucleotide, nicotinamide mononucleotide (NMN), ADP, ATP, GTP, glucose 6-phosphate, yeast alcohol dehydrogenase, horse liver alcohol dehydrogenase, yeast glucose 6-phosphate dehydrogenase and Crotalus adamanteus nucleotide pyrophosphatase were purchased from Sigma. Matrex gel Green A and Matrex gel Blue A were obtained from Amicon. 3-Aminopyridine adenine dinucleotide (AAD) was prepared by a published procedure (Fisher et al., 1973). All other chemicals were of reagent grade.

\section{Results}

\section{Effect of pyridine mononucleotides on growth}

Since earlier studies (Kahn \& Anderson, 1986) of growth of $H$. influenzae had demonstrated that 3-acetylpyridine adenine dinucleotide supported growth of the organism in the absence of NAD and served as a substrate for the $H$. influenzae periplasmic nucleotide pyrophosphatase, it was of interest to investigate the assumed ability of 3acetylpyridine mononucleotide (APMN) to likewise support growth of the organism. The effectiveness of APMN in supporting growth of the organism is shown in Fig. 1, and compared to other pyridine nucleotides in Table 1. At a concentration of $1 \mu \mathrm{g} \mathrm{ml}^{-1}$, the rate of growth on NMN was approximately twice that observed with APMN. Although APMN appears more effective than 3-acetylpyridine adenine dinucleotide (APAD) in support of growth (Table 1), they are equally effective when compared on a molar basis.

The effectiveness of 3-aminopyridine mononucleotide (AMN) as an inhibitor of growth was compared to that previously documented for 3-aminopyridine adenine dinucleotide (AAD) (Kahn \& Anderson, 1986). With NAD as the source of pyridine nucleotide growth factor, both AAD and AMN inhibited growth of $H$. influenzae at submicromolar concentrations (Table 2) and were essentially equally effective on a molar basis. The same relationship was observed in growth inhibition by the 3-

Table 1. Ability of various pyridine nucleotides to serve as $V$-factor for growth of $H$. influenzae

\begin{tabular}{ccc}
\hline Pyridine nucleotide & $\begin{array}{c}\text { Concn. } \\
\left(\mu \mathrm{g} \mathrm{ml}^{-1}\right)\end{array}$ & $\begin{array}{c}\text { Doubling } \\
\text { time (min) }\end{array}$ \\
\hline NAD & 1.0 & 39 \\
APAD & 1.0 & 250 \\
NMN & 1.0 & 54 \\
APMN & 1.0 & 93 \\
APMN & 3.0 & 60 \\
\hline \hline
\end{tabular}

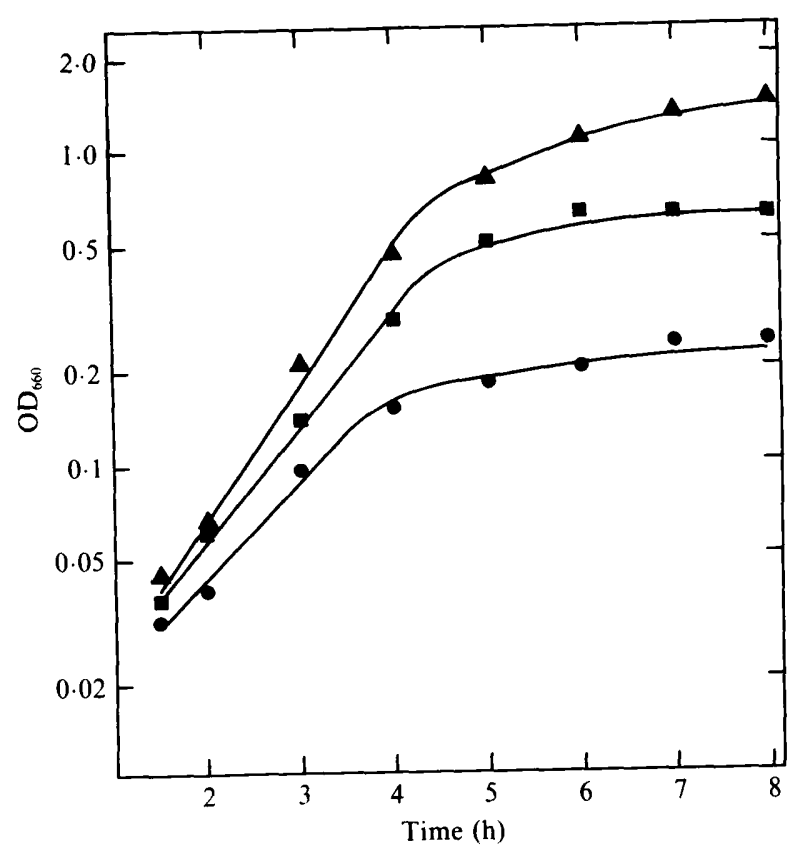

Fig. 1. Growth of $H$. influenzae with APMN as V-factor. The organism was grown as described in Methods. The concentrations of APMN used were: $0.05 \mu \mathrm{g} \mathrm{ml}^{-1}(\mathbf{O}) ; 1 \mathrm{mg} \mathrm{ml}^{-1}(\mathbf{\square})$; and $3 \mu \mathrm{g} \mathrm{ml}^{-1}(\mathbf{\Delta})$.

Table 2. Inhibition of growth of $H$. influenzae with NAD or $N M N$ as $V$-factor $\left(0.1 \mu \mathrm{g} \mathrm{ml}^{-1}\right)$

\begin{tabular}{llcc}
\hline \hline V-factor & Inhibitor & $\begin{array}{c}\text { Concn* } \\
\left(\mu \mathrm{g} \mathrm{m}^{-1}\right)\end{array}$ & $\begin{array}{c}\text { Doubling time } \\
(\mathrm{min})\end{array}$ \\
\hline \multirow{2}{*}{ NAD } & - & - & 65 \\
& AAD & $0.1(0.15)$ & 85 \\
& AAD & $1.0(1.50)$ & NG \\
& AMN & $0 \cdot 1(0.31)$ & 198 \\
NMN & AMN & $1.0(3 \cdot 10)$ & NG \\
& AAD & - & 73 \\
& AAD & $1.1(0 \cdot 15)$ & 135 \\
& AMN & $0.1(0.31)$ & NG \\
& AMN & $1.0(3.10)$ & 270 \\
& ADP & $4.5(10.5)$ & NG \\
& ADP/AAD & $4.5 / 1.0(10.5 / 1.5)$ & 74 \\
\hline \hline
\end{tabular}

NG, No growth observed.

* Numbers in parentheses are concentrations $(\mu \mathrm{mol})$ of inhibitors.

aminopyridine derivatives when NMN was used as the V-factor source. As reported earlier (Kahn \& Anderson, 1986), $1.5 \mu \mathrm{M}$-ADP causes $50 \%$ inhibition of growth of $H$. influenzae on NAD and is the concentration that represents the $K_{\mathrm{i}}$ value for ADP inhibition of the periplasmic nucleotide pyrophosphatase. However, seven times this concentration of ADP had no effect on growth when NMN was used as the pyridine nucleotide source (Table 2). In the presence of this concentration of ADP, which totally inhibits the nucleotide pyrophosphatase, AAD was observed not to inhibit growth on NMN. 


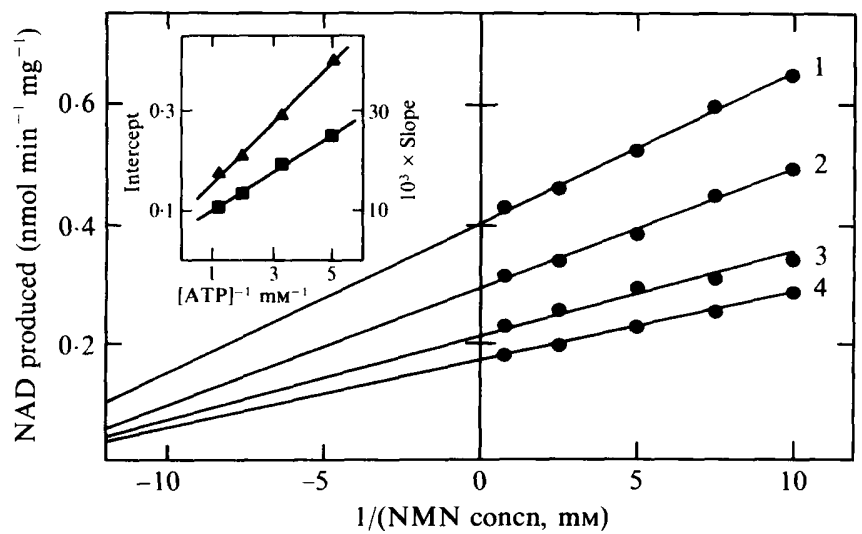

Fig. 2. Effect of NMN and ATP concentrations on NMN adenylyltransferase initial velocities. Assay mixtures contained concentrations of NMN varying from 0.1 to $1.3 \mathrm{mM}$ at four fixed concentrations of ATP in $50 \mathrm{~mm}$-Tris/ $\mathrm{HCl}, \mathrm{pH} 8.0$. The ATP concentrations used were: line $1,0.2 \mathrm{~mm}$; line $2,0.3 \mathrm{~mm}$; line $3,0.5 \mathrm{mM}$, and line $4,0.8 \mathrm{~mm}$. Inset is a replot of slopes $(\mathbf{D})$ and intercepts $(\boldsymbol{\Delta})$.

\section{Studies of NMN : ATP adenylyltransferase}

Since APMN supported growth of the organism and AMN effectively inhibited growth, the interactions of these mononucleotides with the cytoplasmic NMN : ATP adenylyltransferase were investigated. The partially-purified NMN:ATP adenylyltransferase was relatively unstable, losing $30 \%$ activity after $4 \mathrm{~d}$ at $-15^{\circ} \mathrm{C}$. Attempts to stabilize the enzyme with the individual addition of $2 \mathrm{mM}-\mathrm{EDTA}, 10 \%(\mathrm{v} / \mathrm{v})$ propylene glycol, $0.1 \%$ bovine serum albumin, $5 \mathrm{mM}-\mathrm{MgCl}_{2}$ or $0 \cdot 1 \mathrm{M}-\mathrm{KCl}$ failed. However, the partially-purified enzyme in $50 \mathrm{~mm}$-potassium phosphate, $\mathrm{pH} 7 \cdot 5$, containing $0.5 \mathrm{~mm}$-DTT and $1 \mathrm{M}-\mathrm{KCl}$ was stable for 1 week at $-15^{\circ} \mathrm{C}$, and was the preparation used to establish kinetic parameters. It was also noted that NAD synthesis was absolutely dependent on the presence of magnesium ions with the optimum concentration being $30 \mathrm{~mm}$. The effect of NMN concentration on initial velocities of NAD formation is shown in Fig. 2. From the replots of slopes and intercepts (Fig. 2, inset), $K_{\mathrm{m}}$ values of $60 \mu \mathrm{M}$ for NMN and $500 \mu \mathrm{M}$ for ATP were determined. Inhibition of NMN:ATP adenylyltransferase by 3aminopyridine derivatives was investigated using $K_{\mathrm{m}}$ concentrations of NMN and ATP. No inhibition by AMN or AAD was observed at concentrations as high as $250 \mu \mathrm{M}$ and $700 \mu \mathrm{M}$, respectively. It was also found that GTP did not substitute for ATP in the reaction catalysed.

The ability of NMN : ATP adenylyltransferase to use APMN as substrate with ATP to form the corresponding dinucleotide, was studied. Absorbance spectra monitored with time between 300 and $420 \mathrm{~nm}$ demonstrated the formation of a peak having an absorbance maximum at $365 \mathrm{~nm}$ corresponding to reduced APAD. In reaction mixtures not containing the coupled enzyme system, the formation of APAD was confirmed by HPLC. The ability of the NMN:ATP adenylyltransferase to use AMN with ATP to form AAD was demonstrated by HPLC and thin layer chromatography.

\section{Studies of NAD kinase}

Since both APMN and AMN functioned as substrates in reactions catalysed by NMN : ATP adenylyltransferase, the products of these reactions were next studied as substrates for the cytoplasmic NAD kinase to evaluate the possible formation of the corresponding NADP analogues. When the concentration of ATP was varied from 25 to $500 \mu \mathrm{M}$ with $0.4 \mathrm{mM}-\mathrm{NAD}$, substrate inhibition was observed at ATP concentrations above $0.2 \mathrm{mM}$. An apparent $K_{\mathrm{m}}$ of $30 \mu \mathrm{M}$ for ATP was determined from the linear portion of double reciprocal plots of these data. At $0.2 \mathrm{mM}$-ATP and NAD varied from 40 to $125 \mu \mathrm{M}$, an apparent $K_{\mathrm{m}}$ of $55 \mu \mathrm{M}$ was determined for NAD. Under these conditions, APAD was investigated as a substrate for NAD kinase using a wavelength of $365 \mathrm{~nm}$ for assay by the coupled enzyme system. A $K_{\mathrm{m}}$ for APAD of $500 \mu \mathrm{M}$ was determined and the maximum velocity $(V)$ for APAD phosphate formation was twice that observed for the formation of NADP. An NAD kinase reaction with 2 mM-AAD was monitored by both thin layer chromatography and HPLC. No disappearance of AAD or formation of AAD phosphate was observed in these reactions. Since AAD did not function as a substrate for the NAD kinase, its possible role as a substratecompetitive inhibitor was studied. Under the same conditions used for the determination of kinetic parameters, no inhibition of NAD kinase was observed with AAD at concentrations as high as $1 \mathrm{mM}$.

\section{Studies of NMN glycohydrolase}

In preliminary studies of the $H$. influenzae NAD kinase in cell sonicates, some hydrolysis of NAD was observed. Part of the hydrolysis was due to the presence of nucleotide pyrophosphatase; however, when the disappearance of NAD was studied using a cyanide addition assay (Colowick et al., 1951), hydrolysis at the nicotinamide-ribose bond of NAD was also indicated. This latter hydrolytic process was characterized by an initial lag phase. When $10 \mu \mathrm{M}$-ADP was added to these reaction mixtures to inhibit the nucleotide pyrophosphatase, hydrolysis of the ribosidic linkage of NAD was no longer observed, but hydrolysis of NMN was observed and occurred without a lag phase. Thin layer chromatography identified the products of the NMN hydrolysis to 
be nicotinamide and ribose 5-phosphate. It was concluded that the catalytic activity being studied was that of an NMN glycohydrolase and that the originally observed hydrolysis of NAD occurred only when the nucleotide pyrophosphatase was available to convert the NAD to NMN.

\section{Discussion}

Recent studies of growth of $H$. influenzae on NAD (Kahn \& Anderson, 1986) demonstrated the presence of a periplasmic nucleotide pyrophosphatase that catalyses the hydrolysis of NAD to NMN and AMP. The NMN thus produced could be converted intracellularly to the essential NAD and NADP coenzymes in the reactions catalysed by NMN : ATP adenylyltransferase and NAD kinase, respectively. The ability of structural analogues of NAD to support growth of the organism in the absence of NAD correlated well with their functioning as substrates for the nucleotide pyrophosphatase (Kahn \& Anderson, 1986). The support of growth was therefore related to subsequent interactions of the mononucleotides produced. When the organism was grown on NMN, concentraitons of ADP sufficient to completely inhibit the nucleotide pyrophosphatase had no effect on the rate of growth (Table 2), again indicating that growth inhibition by ADP occurs through inhibition of the pyrophosphatase. It was further demonstrated that the support of growth by APAD and inhibition of growth by AAD could be achieved with equal effectiveness by the corresponding mononucleotides, APMN and AMN, respectively (Tables 1 and 2 ). That the growth inhibition by AAD actually occurred through subsequent interactions of the AMN was demonstrated by the lack of growth inhibition by the dinucleotide when $10.5 \mu \mathrm{M}$ ADP was present to inhibit the nucleotide pyrophosphatase (Table 2).

Evidence for the intracellular conversion of pyridine mononucleotides to the corresponding dinucleotides was obtained by demonstrating the mononucleotides to serve as substrates for the NMN:ATP adenylyltransferase. The inherent instability of the transferase and the relatively low concentration of the enzyme in cell sonicates precluded purification beyond the 15-fold achieved here. Similar problems with purification were observed in studies of NMN : ATP adenylyltransferase in Lactobacillus fructosus (Ohtsu \& Nishizuka, 1971), Escherichia coli (Dahmen et al., 1967) and Haemophilus haemoglobinophilus (Kasarov \& Moat, 1973). Attempts to stabilize the $\boldsymbol{H}$. influenzae transferase are currently being made.

In order for 3-acetylpyridine derivatives to support growth of $H$. influenzae in the absence of NAD, the NAD and NADP analogues of 3-acetylpyridine should be available intracellularly. Evidence for the conversion of APAD to APAD phosphate was obtained by demonstrating that the NAD kinase accepted APAD as a substrate. By comparing the $V / K_{\mathrm{m}}$ ratios obtained in NAD kinase reactions, the 3-acetylpyridine analogue was demonstrated to be $22 \%$ as effective as NAD. The 3acetylpyridine analogues of NAD and NADP have been observed previously to function with a variety of dehydrogenases from many different sources. In recent studies of $\boldsymbol{H}$. influenzae malate (Yoon \& Anderson, 1989) and 6-phosphogluconate (Yoon et al., 1989) dehydrogenases purified to electrophoretic homogeneity, the 3acetylpyridine analogues were demonstrated to function effectively as coenzymes.

Although AMN served as a substrate for $H$. influenzae NMN : ATP adenylyltransferase, the resulting dinucleotide did not function as a substrate for the NAD kinase. Therefore, one may be able to rule out the potent growth inhibition observed at submicromolar concentrations of the 3-aminopyridine mono- and dinucleotides as resulting from interactions of the NADP analogue since intracellular synthesis of this derivative would not be expected. The present study shows that the 3-aminopyridine mono- and dinucleotides do not inhibit the NMN : ATP adenylyltransferase or the NAD kinase, and in previous studies, the 3-aminopyridine analogues were not effective inhibitors of the $\boldsymbol{H}$. influenzae malate dehydrogenase (Yoon \& Anderson, 1989) and 6-phosphogluconate dehydrogenase (Yoon et al., 1989). Since AMN can be converted to the corresponding dinucleotide, it remains unclear as to which 3-aminopyridine derivative is more important in the growth inhibition process. In addition to the pyridine nucleotide-dependent enzymes discussed above, the observed presence of NMN glycohydrolase activity represents another possible site for inhibition by 3-aminopyridine derivatives. In Salmonella typhimurium and E. coli NMN glycohydrolase activity was thought to be associated with NMN transport into the cells (Tritz, 1987). If the NMN glycohydrolase of $\boldsymbol{H}$. influenzae is similarly associated with the transport of NMN, it would represent an important possible site for selective inhibition by 3aminopyridine nucleotides which could lead to inhibition of growth of the organism. Studies are currently underway to purify and characterize this NMN glycohydrolase.

These studies were supported by Research Grant DBM 8818529 from the National Science Foundation.

\section{References}

BradFord, M. M. (1976). A rapid and sensitive method for the quantitation of microgram quantities of protein utilizing the principle of protein-dye binding. Analytical Biochemistry 72, 248-254. 
Christ, W. \& COOPER, H. (1980). Preparation and purification of nicotinamide mononucleotide analogs. Methods in Enzymology 66, $71-81$.

Cleland, W. W. (1979). Statistical analysis of enzyme kinetic data. Methods in Enzymology 63, 103-138.

Colowick, S. P., KaPLAN, N. O. \& CiotTI, M. M. (1951). The reaction of pyridine nucleotide with cyanide and its analytical use. Journal of Biological Chemistry 191, 447-460.

Dahmen, W., Webb, B. \& Preiss, J. (1967). The deamido-diphosphopyridine nucleotide and diphosphopyridine nucleotide pyrophosphorylases of Escherichia coli and yeast. Archives of Biochemistry and Biophysics 120, 440-450.

Doern, G. V., Jorgensen, J. H., Thornsberry, C. \& Preston, D. A. (1986). Prevalance of antimicrobial resistance among clinical isolates of Haemophilus influenzae. Diagnosis of Microbial Infectious Diseases 4, 95-107.

FISHER, T. L., Vercellotti, S. V. \& ANDERSON, B. M. (1973). Interactions of 3-amino pyridine dinucleotide with dehydrogenases. Journal of Biological Chemistry 248, 4293-4299.

Givner, L. B., Abramson, J. S. \& WasilausKas, B. (1989). Meningitis due to Haemophilus influenzae type $B$ resistant to ampicillin and chloramphenicol. Reviews of Infectious Diseases 11, 329-334.
GunN, B. A., WoOdall, J. B., Jones, J. F. \& ThornSBerry, C. (1974). Ampicillin-resistant Haemophilus influenzae. Lancet II, 845.

KAHN, D. W. \& ANDERSON, B. M. (1986). Characterization of Haemophilus influenzae nucleotide pyrophosphatase. Journal of Biological Chemistry 261, 6016-6025.

KASAROV, L. B. \& MOAT, A. G. (1973). Biosynthesis of NAD in Haemophilus haemoglobinophilus. Biochimica et Biophysica Acta 320, 372-378.

OHTSU, E. \& NishizUKa, Y. (1971). Nicotinamide phosphoribosyltransferase and NAD pyrophosphorylase from Lactobacillus fructosus. Methods in Enzymology 188, 127-132.

Syriopoulou, V., Scheifele, D., Smith, A. L., Perry, P. M. \& HowIL, V. (1978). Increasing incidence of ampicillin resistance in Haemophilus influenzae. Journal of Pediatrics 92, 889-892.

TRITZ, G. J. (1987). NAD biosynthesis and recycling in Escherichia coli and Salmonella typhimurium. Cellular and Molecular Biology 1, 557-563.

YOON, H. \& ANDERson, B. M. (1989). Site-directed inhibition of Haemophilus influenzae malate dehydrogenase. Journal of General Microbiology 135, 245-250.

Yoon, H., ANDerson, C. D. \& ANDerson, B. M. (1989). Kinetic studies of Haemophilus influenzae 6-phosphogluconate dehydrogenase. Biochimica et Biophysica Acta 994, 75-80. 